\section{Cocaine/Levamisole-Induced Vasculopathy}

\begin{abstract}
A 45-year-old woman was admitted to the hospital with non-inflammatory retiform purpura of the extremities and face that developed shortly after using cocaine. Her laboratory evaluation revealed neutropenia, thrombocytopenia, phospholipid antibodies, antinuclear antibodies, and perinuclear antineutrophil cytoplasmic antibodies. A skin biopsy showed a thrombotic vasculopathy. Based on her constellation of findings, a diagnosis of cocaine/levamisole-induced vasculopathy was rendered. Historically, levamisole has been utilized for its anthelmintic and immunomodulatory properties; it is now widely used as a cocaine adulterant. The classic tetrad of the cocaine/levamisole-induced vasculopathy syndrome includes non-inflammatory retiform purpura involving the cheeks and/or earlobes, neutropenia, ANCA positivity, and evidence of recent cocaine use. The differential diagnosis, clinical approach, etiology, and management of this entity are discussed.
\end{abstract}

\section{KEY WORDS}

Levamisole, cocaine, vasculopathy, purpura, neutropenia, neutrophil cytoplasmic antibodies

\section{Michelle T. Chevalier, August A. Natalie}

Department of Medicine and Division of Dermatology, John $H$. Stroger, Jr. Hospital of Cook County, Chicago, IL 6o612, USA

\section{Correspondence}

August A. Natalie, MD

John H. Stroger, Jr. Hospital of Cook County

1900 W. Polk St.

Administration Building, Room 519

Chicago, IL 60612

Phone: (312) 864-4478

Fax: (312) 864-9663

Email: anatalie@gmail.com

Submitted: March 3, 2012

Accepted: April 2, 2012

\section{Introduction}

This report presents a patient with non-inflammatory retiform purpura of the extremities and face, neutropenia, and perinuclear antineutrophil cytoplasmic antibodies accompanying cocaine abuse.

\section{Case Presentation}

A 45-year-old woman was admitted to the hospital because of acute-onset, painful skin lesions of the extremities and face. The patient had a history of primary ovarian insufficiency and cocaine abuse. She had used intranasal cocaine several times weekly for the last 25 years. The skin lesions began on the lower extremities, and within hours had extended to the upper extremities and face. These lesions started one hour after using "double the usual amount" of cocaine. They were painful and non-pruritic. She recalled similar, albeit less severe, lesions in the past, which resolved spontaneously in two days. She was not taking any medications. Review of systems was notable for chronic arthralgias. She denied alopecia, oral ulcers, chest pain, dyspnea, cough, abdominal pain, hematuria, or a history of spontaneous abortion.

The patient was alert and oriented on examination. She was afebrile, and her vital signs were normal. Large, tender patches of non-inflammatory retiform purpura were present on the lower extremities (Figures 1 and 2). Tense hemorrhagic bullae appeared within the purpuric lower ex-

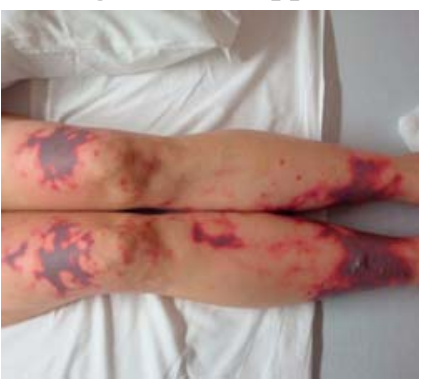

Figure 1. Extensive patches of non-inflammatory retiform purpura on the lower extremities. Hemorrhagic bullae could be seen on the distal, anterior aspect of right lower extremity.

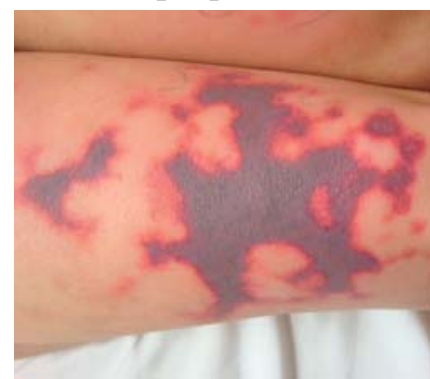

Figure 2. Closer inspection of the right anterior thigh patch showed classic non-inflammatory retiform changes. tremity lesions (Figure 1). The upper extremities, nasal tip, and left lower cheek had similar morphologic changes, but these were less violaceous (Figure 3). The trunk and ears were spared. The remainder of the examination was nor- 
mal. The serum levels of electrolytes, calcium, BUN, creatinine, as well as liver function tests were unremarkable. Her white blood cell count was 1,700 per microliter; the absolute neutrophil count was 700 per microliter, and platelets were 39,000 per microliter. Coagulation studies revealed an isolated prolonged partial thromboplastin time of 108 seconds. The following serum immunologic studies were positive: antinuclear antibodies (titer $>1: 160$, speckled pattern); double-stranded DNA (dsDNA) antibodies; lupus anticoagulant screen; cardiolipin antibodies (143.4 units per milliliter, reference range 0 to 20 units per milliliter); perinuclear antineutrophil cytoplasmic antibodies (pANCA) (titer 1:160); and myeloperoxidase antibodies (25.5 units per milliliter, reference range o to 9 units per milliliter). Plasma levels of fibrinogen and serum $\mathrm{C}_{3}$ and $\mathrm{C}_{4}$ complement were unremarkable. Rheumatoid factor was negative, and there was no evidence of cryoglobulins, hepatitis A, B, and $C$ viruses, or human immunodeficiency virus type $1 / 2$ antibodies. We found no antibodies against neutrophil cytoplasmic proteins (cANCA), proteinase 3 (PR3), or beta-2 glycoprotein 1. Sm (Smith) antibodies, ribonucleoprotein antibodies, SS-A/Ro antibodies, and SS-B/La antibodies were also lacking. Urinalysis was unremarkable. A urine drug screen was positive for cocaine.

While this patient was in the hospital, her skin lesions did not enlarge, nor did she develop any new lesions. She was placed under neutropenic isolation precautions, and dermatology, hematology, and rheumatology services were consulted. On hospital day three, a four millimeter punch biopsy was taken from the edge of the left posterior thigh patch; this revealed findings of thrombotic vasculopathy with epidermal necrosis and hemorrhage (Figure 4). There was no evidence of vasculitis. Accordingly, the diagnosis of cocaine/levamisole-induced vasculopathy was made.

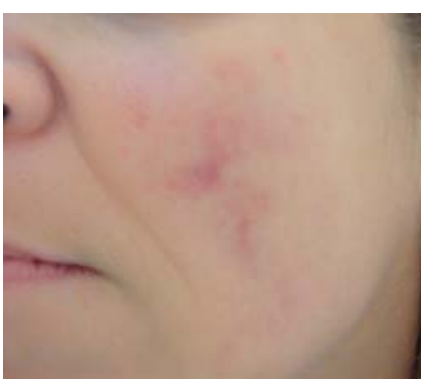

Figure 3. The left lower cheek revealed a faint patch of non-inflammatory retiform purpura.

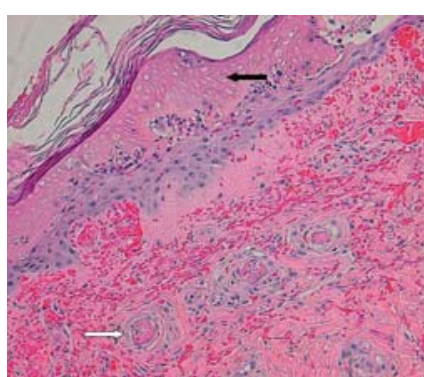

Figure 4. Skin biopsy from the left posterior thigh revealed a thrombotic vasculopathy (white arrow) with epidermal necrosis (black arrow) and hemorrhage (hematoxylin and eosin, $\times 20$ ).
A plasma sample drawn on hospital day three was sent to an outside laboratory for the determination of levamisole. It was reported to be negative eight days later. The patient was discharged in good condition on hospital day five with a recommendation for close outpatient follow up. At the time of discharge, the absolute neutrophil count was 817 per microliter and platelets were 119,000 per microliter. The patient never returned for outpatient care.

\section{Discussion}

The differential diagnosis of non-inflammatory retiform purpura requires concise discrimination. The diagnostic possibilities include all of the cutaneous microvascular occlusion syndromes and the ANCA vasculitides, especially granulomatosis with polyangiitis (Wegener's) and microscopic polyangiitis. A minimal history and physical examination in addition to laboratory findings can quickly separate the diagnostic possibilities of non-inflammatory retiform purpura; however, the details of this diagnostic approach are beyond the scope of this article. The size and number of lesions, as well as the distribution, narrow the possibilities to cryoglobulinemia (particularly type 1), purpura fulminans, catastrophic antiphospholipid syndrome, granulomatosis with polyangiitis (Wegener's), and cocaine/levamisole-induced vasculopathy. Facial retiform purpura, as seen in this case, indicates several possibilities for diagnosis: type 1 cryoglobulinemia, granulomatosis with polyangiitis (Wegener's) and cocaine/levamisole-induced vasculopathy. The unremarkable total protein:albumin ratio, negative rheumatoid factor and cryoglobulin assays, and lack of hepatitis $\mathrm{C}$ viral infection make the diagnosis of cryoglobulinemia (all types) unlikely. Purpura fulminans and catastrophic antiphospholipid syndrome are ruled out by her non-toxic presentation and stable hospital course with lack of widespread large vessel thromboses, multiorgan failure, and disseminated intravascular coagulation. The absence of significant pulmonary and renal involvement rules out granulomatosis with polyangiitis (Wegener's).

Levamisole is an anthelmintic agent that was used in humans for its immunomodulatory properties, including enhanced macrophage chemotaxis and T-lymphocyte function. Additional uses included treatment of rheumatoid arthritis, pediatric nephrotic syndrome, colon cancer, head and neck cancer, and melanoma., ${ }^{1,2}$ The drug was voluntarily withdrawn from the United States market in 1999 due to its adverse side effects.

More recently, in 2002, levamisole was identified in illegally sold cocaine as a substance used to "cut" the cocaine for more profitable sales. The percentage of levamisoleadulterated cocaine has risen steadily since $2006,{ }^{3}$ and it is now found in more than $80 \%$ of the cocaine seized at United States borders. ${ }^{4}$ This adulterant, which alters the brain's metabolism of norepinephrine, dopamine, and serotonin, ${ }^{5}$ is thought to enhance the euphoric and addictive qualities of the cocaine.

The classic tetrad of cocaine/levamisole-induced vasculopathy is defined as non-inflammatory retiform purpura 
involving the cheeks and/or earlobes, neutropenia, ANCA positivity, and evidence of recent cocaine use. The autoantibody profile tends to be solely pANCA positive or both pANCA/cANCA positive; rarely is it only cANCA positive. Antinuclear antibodies and phospholipid antibodies are frequently present. The level of neutropenia is believed to correlate with the ANCA titer. In rheumatology patients treated with levamisole, the presence of HLA B27 may be a risk factor for the development of neutropenia. ${ }^{6}$ Reversible thrombocytopenia and arthritis have also been reported. ${ }^{1}$, 7 There are currently no standardized diagnostic criteria.

Because the half-life of levamisole is 5.6 hours with limited excretion of the parent drug in the urine $(<5 \%)$, a negative test result should be interpreted with caution. Urinary gas chromatography/mass spectrometry (GC/MS) is the preferred method for identification of levamisole. ${ }^{8}$

The etiology of the vasculopathy is not entirely understood, but most experts feel that it results from the development of phospholipid antibodies. There have been multiple reports of vasculitis on histology; $;^{1,2}$ however, we believe that the skin changes are due to microvascular occlusion rather than primary vasculitis. All microvascular occlusion syndromes can present the misleading histologic appearance of vasculitis if an inappropriate site is biopsied, or if the biopsy is delayed.

The vasculopathy will resolve with cessation of cocaine. There is typically no associated long-term sequelae, although a recent study suggested a possible association between idiopathic pulmonary hypertension and levamisole. ${ }^{4}$ Abnormal serologic markers should be followed serially; these abnormalities usually resolve within 2-10 months. ${ }^{9}$ Supportive care is indicated and appropriate. Immunosuppressive therapy, including systemic steroids, should be avoided because of the attendant neutropenia. Filgrastim and sargramostim can be considered if severe neutropenia develops, or if the patient is at high risk for neutropenic complications.

\section{Author contributions}

Both authors contributed equally to this report.

\section{Conflicts of interest}

No potential conflicts of interest relevant to this article was reported.

\section{References}

1. Poon SH, Baliog CR, Jr., Sams RN, Robinson-Bostom L, Telang $\mathrm{GH}$, Reginato AM. Syndrome of cocaine-levamisole-induced cutaneous vasculitis and immune-mediated leukopenia. Seminars in arthritis and rheumatism 2011;41:434-44.

2. Gross RL, Brucker J, Bahce-Altuntas A, et al. A novel cutaneous vasculitis syndrome induced by levamisole-contaminated cocaine. Clinical Rheumatology 2011;30:1385-92.

3. Casale JF, Corbeil EM, Hays PA. Identification of levamisole impurities found in illicit cocaine exhibits. Microgram J 2008;6:82-9.

4. Karch SB, Mari F, Bartolini V, Bertol E. Aminorex poisoning in cocaine abusers. International journal of cardiology $2011 \mathrm{Jul} 15$. [Epub ahead of print].

5. Spector S, Munjal I, Schmidt DE. Effects of the immunostimulant, levamisole, on opiate withdrawal and levels of endogenous opiate alkaloids and monoamine neurotransmitters in rat brain. Neuropsychopharmacology 1998;19:417-27.

6. Hodinka L, Geher P, Meretey K, Gyodi EK, Petranyi GG, Bozsoky S. Levamisole-induced neutropenia and agranulocytosis: association with HLA B27 leukocyte agglutinating and lymphocytotoxic antibodies. Internat Arch Aller Appl Immunol 1981;65:460-4.

7. Winquist EW, Lassam NJ. Reversible thrombocytopenia with levamisole. Med Ped Oncol 1995;24:262-4.

8. Buchanan JA, Heard K, Burbach C, Wilson ML, Dart R. Prevalence of levamisole in urine toxicology screens positive for cocaine in an inner-city hospital. JAMA 2011;305:1657-8.

9. Chung C, Tumeh PC, Birnbaum R, et al. Characteristic purpura of the ears, vasculitis, and neutropenia--a potential public health epidemic associated with levamisole-adulterated cocaine. J Amer Acad Dermatol 2011;65:722-5. 